\title{
11 \\ Migrating from ISODE/ROSE to DCE/RPC: A Common Interface and a Compiler
}

\author{
J. Hummes and W. Gerteis \\ University of Karlsruhe and Digital Equipment GmbH \\ University of Karlsruhe, Institute of Telematics, Postfach 6980, 76128 \\ Karlsruhe, Germany. email: \\ hummes@telematik.informatik . uni-karlsruhe.de \\ Digital Equipment GmbH, CEC Karlsruhe, European Applied Research \\ Center, Vincenz-Prießnitz-Str. 1, 76131 Karlsruhe, Germany. email: \\ gerteis@nestvx.enet.dec.com
}

\begin{abstract}
ROSE of the OSI Reference Model and the RPC paradigm are two major concepts in the area of distributed computing. ISODE and DCE RPC are implementations of these models. This paper is addressed towards distributed application programmers, who use the RO-notation of ASN.1 as specification language and want to develop RPC applications as well.

We introduce an approach of a common interface, which consists of two parts: A common interface to the runtime environments of ISODE and DCE, and a transformation from a specification given in the RO-notation into DCE IDL. Using this interface we provide a migration path from an application based on ISODE to one based on DCE. The concept also enables newly developed applications to run in both environments, which increases the number of potential users substantially. Furthermore the specification of user specific data types and operations can be preserved in ASN.1, since the transformation into DCE IDL is generated by the compiler ro2idl.
\end{abstract}

Keywords

Open distributed systems, ASN.1 compiler, DCE IDL, ROSE, RPC

\section{INTRODUCTION: FOUNDATIONS AND MOTIVATION}

Building distributed applications in a heterogeneous environment needs support by distributed environments. Two well-known concepts about information exchange in those environments are often used at the programming level: the Remote Operations Service Ele- 
ment (ROSE) of the Open Systems Interconnection (OSI) Reference Model (ISO 7498, 1984) and the Remote Procedure Call (RPC) paradigm (Nelson, 1981).

The OSI Reference Model proposes a layered network model with seven layers: The four lower layers provide end-to-end services; the upper three layers provide application services (Tanenbaum, 1989; Rose, 1990). The application layer manages the communications between applications. It differs from the layers beneath by explicitly being divided into service elements (Rose, 1990). The application service elements ACSE (association control) and ROSE (remote operations) are needed to call remote operations. Application contexts, application service elements and its remote operations are specified by using the macro package $R O-$ notation (ISO/IEC 9072, 1989) for the specification language Abstract Syntax Notation One (ASN.1) (ISO 8824, 1987).

The ISO Development Environment (ISODE) (Rose et al., 1992) is an implementation of the higher layers. Given a specification in the RO-notation, distributed applications can be developed supported by two compilers, rosy and pepsy. These compilers generate stubs and type declaration files from the specification with the necessary coding functions in the $\mathrm{C}$ language. Together with its runtime libraries ISODE is a powerful toolkit for building distributed applications based on the OSI model.

The RPC paradigm differs from ROSE in three essential points: First, the RPC paradigm assumes a client/server model in the control flow. Second, the RPC paradigm does not need a connection being established, before a remote procedure can be called. And finally, the RPC paradigm is ideally location transparent, i.e. there is no syntactic difference between a remote and a local procedure call. Semantically, however, the effects of remoteness are not totally hidden.

The implementation of the RPC paradigm is the heart of the heterogeneous OSF Distributed Computing Environment (Lockhart, 1994; OSF, 1992). However, DCE offers much more than RPCs only. "It is a collection of integrated software components that are added to a computer's operating system" (Bever et al., 1993). The Thread Service, which provides a portable implementation of lightweight processes, and the RPC Service are fundamental for all other services. The Cell Directory Service (CDS) supports distributed name management. The Security Service can be used by the RPC service for authentication, authorization, and encryption. Furthermore, DCE offers the Distributed Time Service, the Global Directory Service, and the Distributed File System.

While the OSI model is standardized by the ISO, DCE has become a de-facto standard in the area of distributed computing. In this paper we show that a distributed application can be developed such that it can use both, ISODE and DCE, as underlying communication service. This increases the number of potential users of such an application substantially. The specification in RO-notation is preserved and may be evolved during the development. The corresponding IDL files are generated automatically by the compiler ro2idl. This ensures consistency and decreases the amount of work.

The richness of distributed services in DCE is a good motivation for migrating from ISODE applications to DCE applications. For example, security plays more and more an important role in distributed applications; with DCE security can be achieved easily. The CDS is a powerful service for locating appropriate servers; with CDS a client does not need to know the server's address in advance. A client can bind to a server dynamically.

The remainder of the paper is organized as follows: Section 2 shows a migration path from ISODE to DCE applications and the role of a common interface to the runtime environments. In section 3 , we analyze the differences between remote operations in ISODE 
and remote procedures in DCE. The approach of a common interface shown in section 4 minimizes the work to be done for migration. It helps also to develop applications in both environments. The compiler ro2idl, which is described in section 5 , generates DCE IDL files from a given ASN.1 specification in RO-notation. Finally, in section 6, a short summary and an outlook to further work is given.

\section{MIGRATION PATH}

Assuming that an existing ISODE application should be migrated from ISODE to DCE the following migration path (s. Figure 1) is practicable.
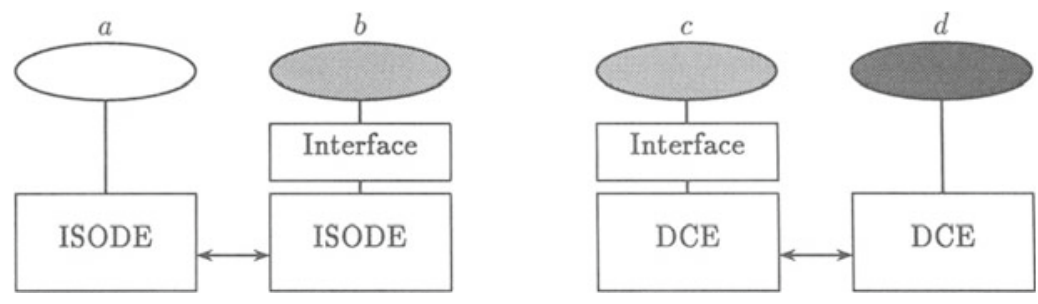

Figure 1 Migration: application uses a) ISODE functions, b) and c) functions of the common interface, d) DCE functions.

First a common interface is designed regarding the guidelines given in the following sections. The invoker and the performer can be evolved separately to use the common interface. When an entity development reaches state (b), it can be tested against (a). Then the environment can be changed from ISODE to DCE and the application (c) can be tested against a pure DCE application (d). Note that the calls to the common interface in (b) and (c) are the same with respect to their syntax and semantics. Only the calls inside the interface have changed in order to use the suitable runtime environment. Having the interface, only a new compilation is needed. State (d) will be reached only in the case that a pure DCE application is desirable and the specification in the RO-notation will not change or is also migrated to a specification in IDL.

The development of a new application will start at (b) or (c). By using the common interface to the runtime environments the application is able to work with ISODE as well as with DCE. Having an application in state (b) or (c) the development of the corresponding application is reduced to the implementation of the interface to its runtime environment. The development of such an interface is well supported by our approach: A design of the runtime part of the interface is presented in the next sections as well as its implementations for ISODE and DCE, which can be put into a library or generated by a compiler. Furthermore, the compiler ro2idl transforms the user specified data types and operations from the RO-notation into DCE IDL. 


\section{ISODE AND DCE APPLICATIONS}

This section discusses similarities and differences between ROSE and RPC on a conceptual level. Understanding of these is fundamental in order to design a common interface and to implement it using ISODE and DCE.

\subsection{Differences between ROSE and RPC}

Distributed programs using the ROSE facilities expect to invoke operations over a logical connection (association). In general, those operations are not designed to be stateless. On the other hand, the RPC paradigm does not require a connection. Therefore, a context between the client and the server has to be established when first calling the server.

The RPC paradigm is founded on the client/server flow model. However, the OSI model is a peer-to-peer architecture. The general case (association class 3) allows operations to be invoked by both application entities. For RPC this means that the server calls back the client. Both, server and client, must be prepared to handle this.

In ROSE, operation invocation, returning the result, and returning an error are three different services. Rejections are possible, too. ISODE maps these services to different runtime function calls. Contrarily, in the RPC paradigm remote procedures are called (ideally) transparent.

Furthermore, remote operations in ROSE are specified inherently asynchronous; ISODE supports both asynchronous and synchronous invocations. In the RPC paradigm procedure calls are synchronous. If an application needs asynchrony, it must be coded in DCE explicitly by using threads.

\subsection{Associations and Bindings}

In OSI, an association between the application entities must be established, before a remote operation can be invoked. Associations are managed by the Association Control Service Element (ACSE). The requesting entity is termed initiator, the accepting entity is termed responder. Each remote operation invocation refers to the established association; in other words: the association forms a context between the application entities.

The semantics of an association should have a corresponding concept in an RPC application. In DCE, they are achieved by the binding methods and the concept of context handles:

The binding method determines how a client is bound to a server. Depending on the application's need the user chooses the appropriate method. Context handles are used to maintain the server's state and to establish a logical association between the client and the server. When the first call is made, the server saves the current state associated with this request and sets a context handle to point to it. The context handle is returned to the client, which then provides the handle on all subsequent calls. The DCE runtime environment is responsible that all subsequent calls are directed to the same server; context handles also have binding information (Lockhart, 1994). With the last call the context handle is reset by the server (assigned to NULL) and returned to the client.

In OSI three association classes are defined that indicate, which entity is the invoker and which is the performer of the operations (Rose, 1990). If both, initiator and responder, invoke and perform operations the $\mathrm{RO}$-specification must be split into two different 
RPC specifications to define which application entity acts as server for which procedures. After the client has bound to the server and called the first operation (i.e. established the context), the server calls back the client and establishes a second context. So, the bidirectional association is mapped to two different bindings with context handles.

\subsection{Invoker and Client}

On an abstract level, an invoker and a client look similar (Figure 2).

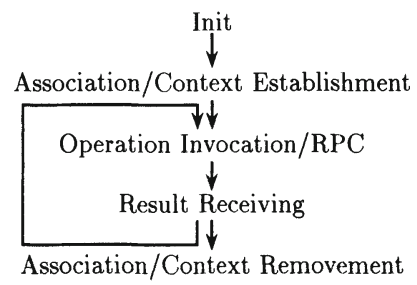

Figure 2 Abstract: Invoker/Client.

After initialization the association to the responder is established. In OSI this is done using the confirmed A-ASSOCIATE service from ACSE. An association identifier is returned. In DCE, the client binds to the server and then calls the first RPC that returns a context handle, which is used in all subsequent calls.

Then remote operations can be invoked using the unconfirmed ROSE service ROINVOKE. Assuming that the operation is well designed with regard to totality, a result or error is sent back later by the responder using one of the unconfirmed services RORESULT or RO-ERROR. The invoker has to react to the service indication to receive the result or error, respectively. Since invocations are asynchronous, it is important that the invoker keeps track of the invoked operations to implement a certain execution semantic. Using ISODE the simplest solution is to use synchronous invocations, which are supported by the library.

In the RPC paradigm, calling a remote procedure is inherently bound to the receiving of its result. DCE allows the user to choose predefined execution semantics.

In OSI an association can be released by both, the initiator and the responder. An orderly release is done by using the confirmed service A-RELEASE. In DCE, the context is released by explicitly calling a remote procedure.

\subsection{Performer and Server}

An abstract view to the tasks of a performer or server is shown in Figure 3. After initializing a performer/server is waiting for service indications or RPCs, respectively. In OSI the application entity is informed by an indication of the underlying ACSE or ROSE services. The indication shows either an association establishment request or an operation invocation. In case of an association establishment request the application entity acts as responder using the ACSE services. In case of an operation invocation, it acts as performer 


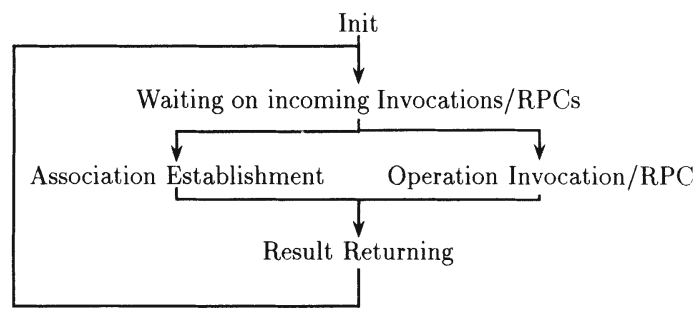

Figure 3 Abstract: Performer/Server.

and using the ROSE services. In both cases, after computing the result it returns it to the requester: A new association is returned by using the A-ASSOCIATION.RESPONSE primitive, return values of an operation are sent back by the RO-RESULT service or the RO-ERROR service, in case of an error.

In DCE, associations are emulated with context handles (s. section 3.2). Establishing and releasing of context handles are done with RPCs. So, no differences between association requests and RPCs must be considered in the structure of the server. Of course, the context handles have to be managed. The RPC behaves like a local procedure.

\section{A CONCEPT FOR A COMMON INTERFACE}

The main task of any interface is information hiding. In our case, the application should not be aware of the underlying communication mechanism.

Our approach of a common interface consists of two parts: An interface to the runtime environments, and one to the specification of the remote operations. As common specification language we choose the RO-notation of ASN.1. Its transformation into DCE IDL is described in section 5 .

In this section, we line out a common interface to the runtime environments of ISODE and DCE. Some examples will illuminate our concept.

\subsection{Data types}

The compilers rosy and pepsy belong to ISODE. Given a RO-specification, they generate the corresponding type declarations and stubs in $\mathrm{C}$. The data types generated by rosy/pepsy differ from the data types generated by ro2idl/idl. Furthermore, application data may be specified differently. Prior to defining a common interface to the runtime environments, it has to be decided which data types should be taken.

Figure 4 shows the relationships between the generated data types by rosy/pepsy and ro2idl/idl and the application specific data types. Since both kinds of the generated types are known, conversion routines between ISODE types and DCE types can be generated automatically. However, the application itself is responsible for converting application specific data to ISODE or DCE data. If an application directly uses one of the generated types, no additional conversion is required. In our approach we assume that an appli- 


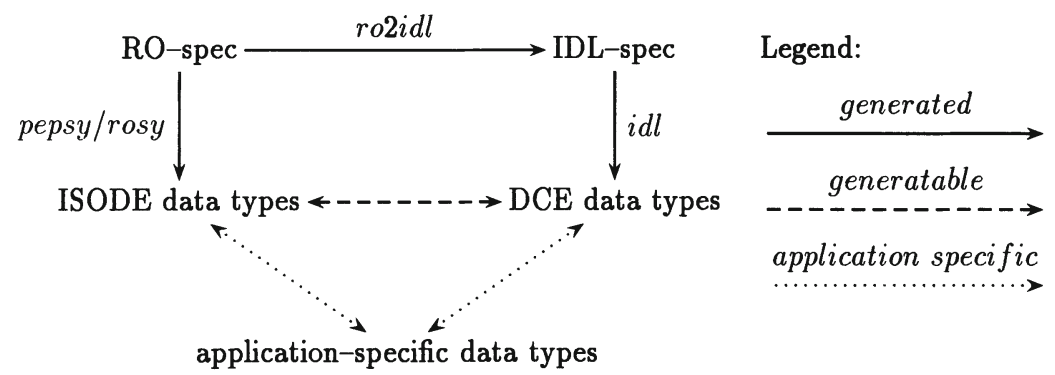

Figure 4 The relationships between the data types.

cation works on ISODE types (i.e. the types generated by rosy/pepsy); the conversion between ISODE data and DCE data is done transparently at runtime using the generated conversion routines.

\subsection{Association control}

In OSI an association must be established, before remote operations can be invoked. This association is referred to by each invocation. In ISODE each association is assigned an association descriptor of type int. Since associations can be modelled with context handles in DCE, a function to map association descriptors to context handles $(\operatorname{sd2ctx}())$, and vice versa $(\operatorname{ctx} 2 \mathrm{sd}())$, belongs to the interface.

Each initiator or client must identify the responder or server it wants to contact, and then connect to it. Setting up the connection is application dependent. Only the association descriptor is of interest for the functions called subsequently by the application; in the example below, the function bind_and_associate() is implemented for ISODE and DCE and returns an integer, which is used as association descriptor or as index to the context handle.

Example 1 This and the following examples are given in pseudo code. On the left side, the ISODE specific code is given, on the right side, the DCE code. The function bind_and_associate():

Identify the responder and set all needed parame- Bind with an appropriate method to the server. Fiters. Finally call

AcAssocRequest (...)

that corresponds to the REQUEST and on successful return to the CONFIRMATION primitive of the A-ASSOCIATE service. Return the association descriptor (sd). nally call the first RPC

setup_context (...)

to get the context handle $(\operatorname{ctx})$ and convert it to an sd:

$s d=\operatorname{ctx} 2 s d(\operatorname{ctx})$;

return( sd );

The responder or server initializes itself and then is capable to respond to association indications or to perform RPCs. Again, initializing is application dependent. At the end of this part the routine is listening and waiting for incoming calls. Depending on the kind of call (association or operation invocation) the routine behaves differently in ISODE. In DCE always the called procedure is performed. 
Since associations are bidirectional the ISODE specific part sets an association descriptor. In DCE the initialization is done in the server control part, while the association control is performed by the called remote procedure, e.g. setup_context (), which stores the context and returns a handle that is associated with a descriptor. If the application has to call RPCs at the client side, the server also calls bind_and_associate(). The client's address can be determined by a DCE runtime function call or is maintained in the setup_context() RPC by the client.

\subsection{Operations}

Although operations in ROSE are asynchronous, the ISODE library supports synchronous invocations. Our paper only points out how a function, which supports both, synchronous ISODE invocations and DCE RPC, looks like.

In ISODE operations are referred to by their operation values. In DCE an RPC is done the same way a local procedure is called. Also the representation of the used data types differs, as mentioned earlier. The simplest way to achieve the common interface, which can use both, the ISODE and the DCE concept, is to encapsulate all possible RPCs into separate functions and to use an operation table. In these functions the data conversion from ISODE to DCE and vice versa is performed, too. A function call_operation() in the common interface should have parameters for the association descriptor, the input, output and error arguments, and a pointer to the called operation.

Example 2 The prototype declaration of the function call_operation().

\begin{tabular}{|c|c|c|}
\hline $\begin{array}{l}\text { all_operation } \\
\qquad \text { int } \\
\text { int } \\
\text { caddr_t } \\
\text { caddr_t } \\
\text { int } \\
\text { caddr_t }\end{array}$ & $\begin{array}{l}\text { op, } \\
\text { sd, } \\
\text { in, } \\
\text { *result, } \\
\text { *errnum, } \\
\text { *errparm) }\end{array}$ & $\begin{array}{l}\rightarrow \text { value of the operation to be invoked } \\
\rightarrow \text { association descriptor } \\
\rightarrow \text { operation's input parameter (ISODE format) } \\
\leftarrow \text { operation's result (ISODE format) } \\
\leftarrow \text { operation's error value, only set if the outcome is an error } \\
\leftarrow \text { operation's error parameter (ISODE format) }\end{array}$ \\
\hline
\end{tabular}

Example 3 Implementation of call_operation() in pseudo code: reduced to the parameters of interest; “..." indicates an omission. The DCE part selects the RPC corresponding to the operation identifier by a switch/case statement. In practice an operation table would be consulted. Let op point to the operation testExample1 with its value testExample1_val.

The arguments sd, op, and in are given directly to switch (op) \{

the function

st $=$ RyOperation $(\mathbf{s d}, \ldots$

op, in, \&out, \&response, ....)

out and response are return values. The opera-

tion returns $\mathrm{OK}$, if no failure occurred. In case of receiving an error response holds the error code, RY_RESULT otherwise.

case testExample1_val:

Convert the ISODE type in to the DCE type arg:

ID_testExample1( (arg, in);

$\operatorname{ctx}=\operatorname{sd} 2 \operatorname{ctx}(\mathrm{sd})$;

Do the RPC:

testExample1 (ctx, arg,

\&out, \&err); 


\section{A COMPILER FROM THE RO-NOTATION TO DCE IDL}

A compiler from the RO-notation to DCE IDL has to support at least the transformation of basic and user defined types, error types, and operations; in short: to handle all the basic RO-notation macros. Furthermore, it has to supply conversion routines between the ISODE and DCE data for migration.

\subsection{Generated Files}

The RO-notation is used to define types and operations, but also to identify which application entity invokes which operations over a bidirectional association. Since DCE RPC is a client/server model, the RO-specification is split by the compiler into four IDL files (Figure 5).

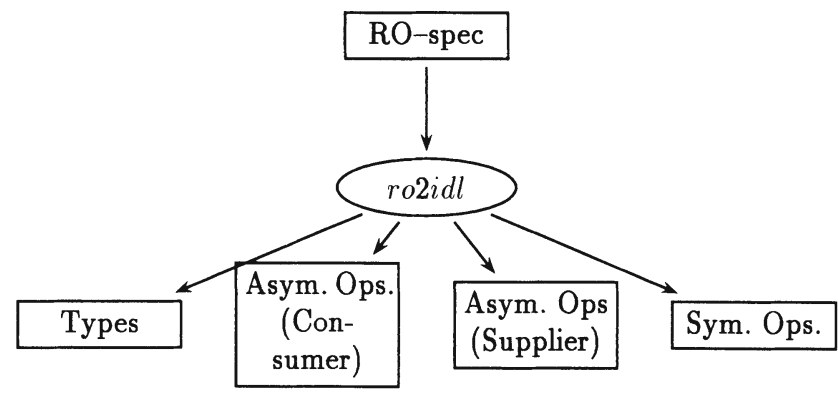

Figure 5 Input and output files of ro2idl.

One file contains all specified types and constants, and one all operations for the symmetric application service elements (ASE). The remaining files comprise the operations for the asymmetric ASEs; one contains all operations performed by the consumer, the other contains all operations performed by the supplier. Therefore, the compiler uses the informations given in the macros APPLICATION-CONTEXT and APPLICATION-SERVICE-ELEMENT. The type definition file is included by all other generated files.

\subsection{Identifier Conversion}

Since IDL does not allow the use of the character "-" in identifiers, each occurrence of that character is mapped to "--". If specified as compiler option each user defined type and operation is preceded by the module name and ".".

\subsection{Type Conversion}

Types are assigned and constructed in the RO-notation using the plain ASN.1 notation. A type can be assigned values or defined by referring other, basic or constructed, types. Only types, which refer to other types are supported in our approach. 
Table 1 Conversion of ASN.1 basic types

\begin{tabular}{llll}
\hline ASN.1 basic type & IDL type & definition in asn1_base.idl \\
\hline IA5String & -IA5String & typodef [string] char* _IA5String; \\
BOOLEAN & -BOOLEAN & typedef boolean & -BOOLEAN; \\
INTEGER & -INTEGER & typedef long & -INTEGER; \\
REAL & -REAL & typedef double & -REAL; \\
\hline
\end{tabular}

Example 6 Assume in module EXPL is a reference to My-Type. Depending on the compiler option, the conversion is as follows:

My-Type $\Rightarrow$ My__Type or expl_My__Type

The conversions of the supported basic types are listed in Table 1. In the first column the ASN.1 types are listed, in the second their mapping in the generated code, and in the last their default type definition in the file asn1_base.idl. This IDL file is included by each generated IDL file; this way it is easy to customize the mapping. The type IA5String is actually no basic type, but a character string type. No other character string type is supported by the compiler at this time.

The ASN.1 type ANY is mapped currently to a union of basic types. It is in the responsibility of the user to change this mapping. The ASN.1 type NULL is handled as a special case. If it occurs in a choice arm or as operation parameter (s. below) it is ignored.

Simple type assignments in the $\mathrm{RO}$-notation are converted to simple type definitions in IDL.

Example 7 A simple type assignment.

YesNo : := BOOLEAN $\quad \Rightarrow \quad$ typedef _BOOLEAN YesNo;

User defined nested types are first de-nested on ASN.1 level and then transformed. For each type implicitly defined by an inner type generator, an explicit type assignment with a unique identifier at the level of the module is created. The inner type generators are then replaced by type references to the new created types.

An ENUNERATED type is converted to an enum type. The values of the elements are ignored, since IDL does not allow assignments to elements. A SEQUENCE type is converted to a struct type. A SEQUENCE OF type is converted to a linked list. The list is built by a struct with two elements. One has the converted ASN.1 type as type, the other element is a pointer to this structure. Additionally a type definition is created: A full pointer to that structure with the name of the ASN.1 tag as identifier; it is used then to refer to the linked list. A CHOICE type is converted to two IDL types, an enum and a union type. The enumeration includes all possible cases. The arms of the union are preceded by mnemonic case values, i.e. the enumeration elements.

A type, which is marked as OPTIONAL, is converted to a full pointer to the corresponding IDL type, i.e. with the attribute ptr. This pointer is assigned NULL, when it is not used.

Example 8 A complexer example shows the compiler's conversion rules. 


\begin{tabular}{|c|c|c|}
\hline Example & $::=$ CHOIC & CE \{ \\
\hline & is-true & [0] YesNo, \\
\hline & int-list & [1] SEQUENCE OF INTEGER, \\
\hline & strange & [2] SEQUENCE \{ \\
\hline & & $\begin{array}{l}\text { string IA5String, } \\
\text { number INTEGER OPTIONAL }\end{array}$ \\
\hline & $\begin{array}{l}\text { \}, } \\
\text { empty }\end{array}$ & [3] NULL \\
\hline$\}$ & & \\
\hline
\end{tabular}

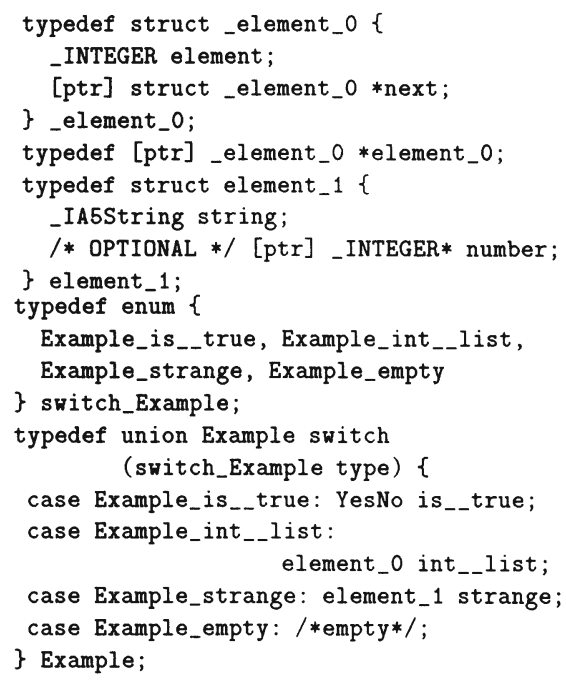

First, the defined type Example is de-nested; YosNo is a type reference and is preserved, the implicit type definitions int-list and strange are declared outside the union in the IDL file with generated names. In the union they are referenced by their new names. The type int-list is a SEQUENCE OF and is therefore mapped to the linked list_element_O. This list is referenced later only by the generated pointer type element_ 0 . The conversion of an optional parameter is shown in the strange sequence with the optional element number. This sequence is mapped to the corresponding IDL structure element_1.

Error types are defined in the RO-notation using the ERROR macro. Each error is assigned a unique value. The error types are referenced in the operation declarations: Each operation has a list of all possible errors. So, the generation of appropriate error types has two steps: First, the error values are mapped to constant declarations in IDL. If the error has a parameter, its type is converted as usual with a new identifier. Then, for each operation an error union is created. Each possible error becomes a union arm. Since the union is only set, if the operation's outcome is an error, it is referenced by a full pointer, which is assigned NULL, if no error occurs.

Example 9 The operation testExample1 may have the two errors exampleError and simpleError.

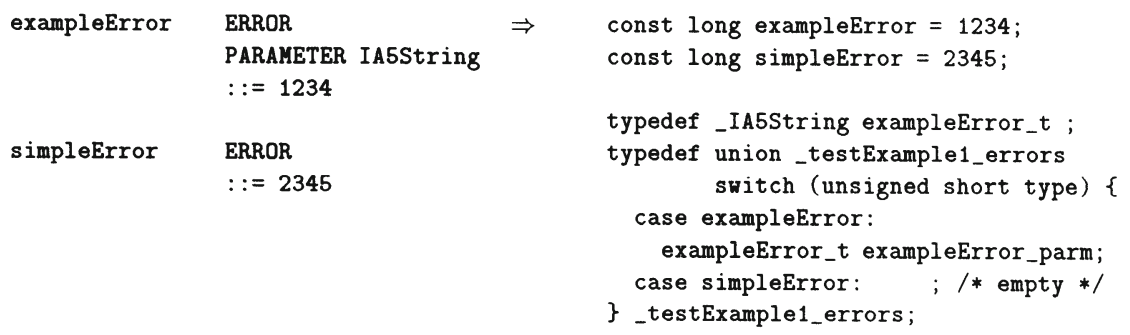




$$
\begin{array}{r}
\text { typedef }[\mathrm{ptr}] \text { _testExample1_errors } \\
\text { *testExample1_errors; }
\end{array}
$$

\subsection{Operation Conversion}

Operations are transformed straightforward. Only type references are allowed as argument and result parameters; value assignments are not supported by our approach. The type reference from the ARGUMENT clause is converted to the type of the input parameter, the parameter's name is always arg. The RESULT clause is handled in a similar way; since result and err are output parameters, they are declared as reference pointers. If the argument or result clause refers to the NULL type, the corresponding parameter declaration is omitted in the IDL file. The ERRORS clause is used also for constructing the error types. In case of an error the err parameter points to the error union; it is assigned NULL, if no error occurred.

Example 10 The types used by the operation are declared in the last examples. The first parameter in the generated RPC is a context handle. Its use is shown in the sections 5.5 and 3.2.

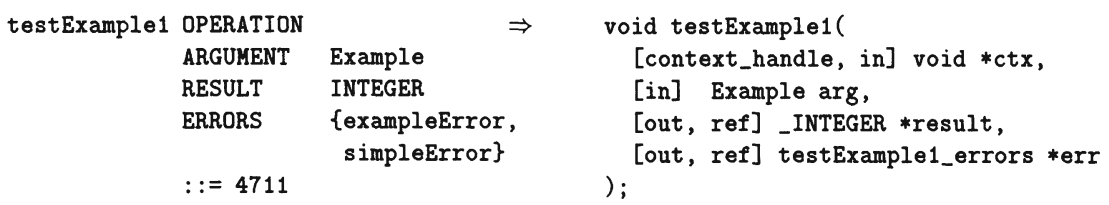

\subsection{DCE-Specific Types}

Before remote operations in the OSI model can be invoked, an association between the application entities must be established. The association is referred in each invocation. In DCE the semantics of an association is achieved by the concept of context handles (s. also section 3.2). For this purpose each RPC is generated with a context handle as first argument.

Two additional RPCs belong in the IDL file: One for the context establishment and one for its release. Since these procedures can differ in respect of binding methods and additional parameters (e.g. a parameter to carry additional binding information), they are included by the programmer.

\section{CONCLUSIONS}

In this paper we presented a migration path from ISODE to DCE. It consists of two parts: A common interface to the runtime environments and a tool for converting a ROspecification of data types and operations to a DCE IDL specification, the compiler ro2idl. The concept of the common interface is a foundation to develop distributed applications in a way that they run in both environments.

Currently, the compiler ro2idl exists as prototype. It is planned to enhance it such that the conversion routines, which are needed in the runtime environment of the common 
interface, are also generated. The compiler will accept all basic ASN.1 types in the near future.

In order to validate the proposed approach, we will use our concept to migrate the software components of the BERKOM MMC-Project (Altenhofen et al., 1993) from ISODE to DCE (Hummes, 1996). As already pointed out, the concept is not confined to ISODE and DCE. It is also valid for other implementations of ROSE or RPC. In the MMCProject another compiler from the RO-notation to XDR (Bloomer, 1992) is currently used (Gerteis, 1995).

\section{REFERENCES}

Altenhofen, Dittrich, Hammerschmidt, Herrtwich, Käppner, Kruschel, Kückes, Steinig (1993) The BERKOM Multimedia Collaboration Service. ACM Multimedia 93.

Bever, Geihs, Heuser, Mühlhäuser, Schill (1993) Distributed Systems, OSF DCE, and Beyond, in DCE - The OSF Distributed Computing Environment, International DCE Workshop, Karlsruhe (ed. A. Schill), Springer Verlag, Berlin Heidelberg, 1993.

Bloomer, J. (1992) Power Programming with RPC, O'Reilly and Associates, Sebastopol.

Gerteis, W. (1995) Transformation of RO-notation to XDR Working paper, CEC Karlsruhe, European Applied Research Center.

Hummes, J. (1996) Ein Abstraktionskonzept für ROSE und RPC auf Basis der Spezifikationssprache ASN.1, Diplomarbeit, Universität Karlsruhe.

ISO 7498 (1984) Information processing systems - Open Systems Interccnnection - Basic Reference Model.

ISO 8824 (1987) Information processing systems - Open Systems Interconnection - Specification of Abstract Syntax Notation One (ASN.1).

ISO/IEC 9072-1 (1989) Information processing systems - Text communication - Remote Operations - Part 1: Model, notation and service definition.

Lockhart, H.W. (1994) OSF DCE: guide to developing distributed applications, McGrawHill, New York.

Nelson, B.J. (1981) Remote Procedure Call, PhD-Thesis, Carnegie-Mellon University.

Open Software Foundation (1992) OSF DCE Application Development Guide, Cambridge.

Rose, M.T. (1990) The Open Book: a practical perspective on OSI, Prentice-Hall, Englewood Cliffs.

Rose, Onions, Robbins (1992) The ISO Development Environment, Vol. 1-4, Version 7.0. Tanenbaum, A.S. (1989) Computer Networks, 2. ed., Prentice--Hall, Englewood Cliffs.

\section{BIOGRAPHY}

Jakob Hummes is studying Computer Science at the University of Karlsruhe. This paper is an extract of his diploma thesis. Wolfgang Gerteis received his Diploma in Computer Science from the University of Karlsruhe in 1989. He then joined the Institute of Telematics of the University of Karlsruhe where he finished his PhD in the area of SW design method support in 1994. Since then he is working in the BERKOM Multimedia Collaboration Project at the CEC Karlsruhe which is Digital Equipment's European Applied Research Center. 